\title{
Red supergiants as supernova progenitors
}

\author{
Ignacio Negueruela ${ }^{1}$, Ricardo Dorda ${ }^{1}$, Carlos González-Fernández ${ }^{2}$ \\ and Amparo Marco ${ }^{1}$ \\ ${ }^{1}$ Departamento de Física, Ingeniería de Sistemas y Teoría de la Señal \\ Escuela Politécnica Superior, Universidad de Alicante \\ Carretera de San Vicente del Raspeig s/n, E03690, San Vicente del Raspeig, Alicante, Spain \\ email: ignacio.negueruela@ua.es \\ ${ }^{2}$ Institute of Astronomy, University of Cambridge, Madingley Road, Cambridge CB3 0HA, \\ United Kingdom
}

\begin{abstract}
Recent searches for supernova II-P progenitors in external galaxies have led to the identification of red objects with magnitudes and colours indicative of red supergiants, in most cases implying quite low luminosities, and hence masses. Current results would suggest that all explosions come from objects less massive than $\sim 18 M_{\odot}$ and that some explosions come from stars with masses well below $10 M_{\odot}$. Stellar models, on the other hand, can only produce explosions from objects more massive than $>9 M_{\odot}$. What does our knowledge of local red supergiants tell us about the expected properties of such objects? We present the results of complementary large spectroscopic surveys and detailed studies of open clusters that point to substantially different observational characteristics depending on metallicity.
\end{abstract}

Keywords. open cluster and associations: general, stars: massive, supergiants, evolution

Red supergiants (RSGs) are detected as progenitors of type II-P supernovae (SNe) and also believed to be the progenitors of other types of SNe (e.g. Smartt 2015). The properties derived for the observed progenitors depend strongly on the way in which observations (essentially, a poorly sampled - and perhaps not single-epoch - spectral energy distribution) are mapped (via stellar evolutionary models) on to fundamental stellar parameters (i.e. temperature and luminosity, and hence mass). The details are complex (references in Smartt 2015), and further complicated by the effects of interstellar and, more to the case, circumstellar extinction (Walmswell \& Eldridge 2012; Kochanek et al. 2012). It is therefore fair to ask ourselves if the properties of RSGs in the Local Universe can give us some insight into the sort of progenitors that we should expect to see.

Given the rarity of RSGs, two complementary approaches can be taken to investigate their properties. The first one is generating large samples of RSGs to characterise galaxywide populations (e.g. González-Fernández et al. 2015). The second approach is to find clusters that are massive enough to contain a large population of coeval RSGs (e.g. Marco et al. 2014). What do we learn from these studies?

Most importantly for progenitor detection, the overall aspect of RSGs depends heavily on metallicity. We find little to no measurable difference in the distribution of spectral types between the Milky Way and the LMC, but the situation changes dramatically for the SMC. In this latter galaxy, the distribution of spectral types is centred on early $\mathrm{K}$ types, and extends into late $\mathrm{G}$ types. In contrast, in the LMC, the distribution of spectral types peaks around M1, extending from mid K to mid M, while in Milky Way clusters most RSGs have types between M1 and M2. This different distribution implies that the amount of dust around a RSG depends strongly on its metallicity, because dust is preferentially seen in M types (e.g. González-Fernández et al. 2015). Moreover, there 
is strong evidence indicating that the brightest RSGs tend to have later spectral types and larger dust envelopes both at LMC and Milky Way metallicities.

This behaviour can be explained in two ways: i) more massive RSGs have a tendency to display later spectral types and lose more mass or ii) RSGs of a given mass evolve from a phase of lower luminosity and mass loss to a phase of higher luminosity and later spectral types. Even though data from Milky Way open clusters seem to favour the second option (or a combination of both), the most massive RSGs in the LMC and the Milky Way do certainly tend to display late ( $\geqslant M 4$ ) types, occupying positions in the HR diagram that are not reproduced by current evolutionary tracks. In contrast, the most luminous RSGs in the SMC do not seem to evolve generally to such late spectral types, and do not present large dust envelopes (Dorda et al., in prep.). They could, on the other hand, be subject to larger spectroscopic variability. If a substantial fraction of RSGs at solar metallicity pass through a phase of heavy mass lost and large circumstellar extinction, their detection rate as progenitors and their average masses could well be underestimated.

\section{References}

González-Fernández, C., Dorda, R., Negueruela, I., \& Marco, A. 2015, A\&\&A, 578, A3

Kochanek, C. S., Khan, R., \& Dai, X. 2012, ApJ, 759, 20

Marco, A., Negueruela, I., González-Fernández, C., et al. 2014 A\&A, 567, A73

Smartt, S. J. 2015, PASA, 32, 16

Walmswell, J. J. \& Eldridge, J. J. 2012, MNRAS, 419, 2054 